\title{
Strong pairing approximation in comparison with the exact solutions to the pairing Hamiltonian
}

\author{
A.V. Lunyov ${ }^{1, a}$ and V.M. Mikhajlov ${ }^{1}$ \\ ${ }^{1}$ Saint-Petersburg State University, Saint-Petersburg, 198504, Russia
}

\begin{abstract}
Results of the Strong Pairing Approximation (SPA) as a method with the exact particle number conservation are compared with those of the quasiparticle method (QM). It is shown that SPA comes to the same equations as QM for the gap parameter, chemical potential and one- and two-quasiparticle states. Calculations are performed for ${ }_{64}^{148} \mathrm{Gd}_{84}$ as an example, and compared with the exact solutions to the pairing Hamiltonian.
\end{abstract}

\section{Introduction}

Investigations of the nuclear superfluidity were initiated to a large extent by the first works of V. G. Soloviev in which the quasiparticle method (QM) was elaborated as applied to the Bardeen-Cooper-Schriffer (BCS) Hamiltonian, $H_{\mathrm{BCs}}$. These works were represented in his well known book [1] where other methods taking into account the conservation of particle numbers in the nuclear pairing more precisely were also described. Several methods mentioned in [1] do not lose significance up to now. Among them it is worth noting methods producing the exact solutions to $H_{\mathrm{BCS}}$, especially the Richardson method applied now to superconducting metal cluster and reproduced in detail in [2], the particle number projection, the Nogami method. Description of other methods can be found in Ref. [2].

One of the ways to allow for invariability of particle numbers in the nuclear pairing is the Strong Pairing Approximation (SPA) suggested in Ref. [3]. This method is based on the experimental fact of a prevailing the monopole pair transfer between ground states of eveneven nuclei with developed pairing over pair transfer to excited states [1]. The same situation is proposed in bulk superconducting systems that were used in the Green function method by Gorkov [4]. Therefore this method was applied in Ref. [3] with taking account of that the particle number is exactly conserved in each state. Additionally, to obtain a simple and closed equation system SPA supposes that seniority-one eigen functions of $H_{\mathrm{BCS}}$ are proportional to functions arising at addition of a particle in a state $t$ to the $N$-particle ground states ( $N$ is even) or at removal of a particle from a state $t$ of the $(N+2)$-particle ground states, that also can take place at a rather strong pairing. More strictly this assumption will be formulated in Sec. 3. We denote the particle number by letter $N$ though all results

\footnotetext{
a e-mail: lunars@mail.ru
}

are applied to both neutron and proton systems with developed pairing.

On this base SPA leads to two equations practically coinciding with equations for the chemical potential $\lambda$ and pairing gap $\Delta$ of the QM [1] though in the SPA these quantities take on another sense. In QM $\lambda$ is a Lagrange multiplier to provide for equality of the expectation value of $N$ with its exact value, while in the SPA it is a measurable quantity, e.g. for $N$-particle system, where $\lambda=[E(N+2)-E(N)] / 2, E(N), E(N+2)$ being the ground state energies of $N$ - and $(N+2)$-systems. The gap parameter $\Delta$ is proportional also to a measurable quantity, the pair transfer matrix element (PTME) between $N$ - and $(N+2)$-ground states, that corresponds to the Gorkov's definition [4]. The quasiparticle energies entering equations for $\lambda$ and $\Delta$ are treated in the SPA as pair energies i.e. as differences $E_{t}(N+1)$, the energy of such seniority-one state in which an odd particle occupies single-particle level $t$, and half of the sum $[E(N+2)+E(N)]$.

Several equations in Ref. [3] follow from commutators of creation and annihilation operators with $H_{\mathrm{BCS}}$. Such a way for deducing diverse formulae is called the equationsof-motion method (EOMM) [5]. Below we show that consistently conducting this method and taking advantage of the foregoing approximations all results of [3] can be obtained, i.e. EOMM in the given case turns out to be equivalent to the Green function method. The EOMM was employed in Ref. [6] for treatment of the nuclear pairing and, in particular, of $s=1$ states ( $s$ is the seniority). In [6], wave functions of these states comprised several $s=0$ states of even systems (up to 8) that required calculations of the corresponding PTME, not only between the ground states. However, the complication of such approach, in our opinion, is comparable with the direct diagonalization of $H_{\mathrm{BCS}}$. The SPA cannot claim to such accuracy as it does not consider admixtures of excited $s=0$ states, though, as it follows from [3] and our results given below, such admixtures in the case of the developed pairing give rather small 
changes in $s=1$ state energies of the order of the pairing strength $g$ (see below in Sec. 4).

\section{Definitions of $\lambda, \Delta$ and quasiparticle energies in SPA}

The standard formulae of the QM are listed, e.g., in Ref. [1]

$$
\begin{gathered}
H_{\mathrm{BCS}}=\sum_{t} \varepsilon_{t} \hat{n}_{t}-g P^{\dagger} P, \\
\hat{n}_{t}=a_{t}^{\dagger} a_{t}+a_{\bar{t}}^{\dagger} a_{\bar{t}}, P^{\dagger}=\sum_{t} a_{t}^{\dagger} a_{\bar{t}}^{\dagger}, \\
\Delta=g\left\langle\Psi_{\mathrm{BCS}}\left|P^{\dagger}\right| \Psi_{\mathrm{BCS}}\right\rangle=g\left\langle\Psi_{\mathrm{BCS}}|P| \Psi_{\mathrm{BCS}}\right\rangle, \\
\left(\begin{array}{l}
u_{t}^{2} \\
v_{t}^{2}
\end{array}\right)=\frac{1}{2}\left[1 \pm \frac{\tilde{\varepsilon}_{t}}{\left(e_{t}\right)_{\mathrm{BCS}}}\right], \\
\tilde{\varepsilon}_{t}=\varepsilon_{t}-\lambda_{\mathrm{BCS}}-g v_{t}^{2},\left(e_{t}\right)_{\mathrm{BCS}}=\sqrt{\tilde{\varepsilon}_{t}^{2}+\Delta^{2}}, \\
\frac{2}{g}=\sum_{t}\left(e_{t}\right)_{\mathrm{BCS}}^{-1}, N=\sum_{t}\left[1-\frac{\tilde{\varepsilon}_{t}}{\left(e_{t}\right)_{\mathrm{BCS}}}\right] .
\end{gathered}
$$

If there is a degeneration in $\varepsilon_{t}$ each term in (5) has to be multiplied by number of pairs on level $t$.

The starting point of the SPA is the exact commutators

$$
\begin{gathered}
{\left[H_{\mathrm{BCS}}, a_{t}^{\dagger}\right]=\left(\varepsilon_{t}-g\right) a_{t}^{\dagger}-g a_{\bar{t}} P^{\dagger},} \\
{\left[H_{\mathrm{BCS}}, a_{\bar{t}}\right]=-\varepsilon_{t} a_{\bar{t}}-g a_{t}^{\dagger} P .}
\end{gathered}
$$

In SPA these commutators are used to ascertain connection between one particle transfer matrix elements $\left\langle(N+1)_{t}\left|a_{t}^{\dagger}\right| N\right\rangle$ and $\left\langle(N+1)_{t}\left|a_{\bar{t}}\right| N+2\right\rangle$

$$
\begin{gathered}
{\left[E_{t}(N+1)-E(N)\right]\left\langle(N+1)_{t}\left|a_{t}^{\dagger}\right| N\right\rangle} \\
=\left\langle(N+1)_{t}\left|\left(\varepsilon_{t}-g\right) a_{t}^{\dagger}-g a_{\bar{t}} P^{\dagger}\right| N\right\rangle, \\
{\left[E_{t}(N+1)-[E(N+2)]\left\langle(N+1)_{t}\left|a_{\bar{t}}\right| N+2\right\rangle\right.} \\
=\left\langle(N+1)_{t}\left|-\varepsilon_{t} a_{\bar{t}}-g a_{t}^{\dagger} P\right| N+2\right\rangle .
\end{gathered}
$$

Here, $|N\rangle,|N+2\rangle$ and $\left|(N+1)_{t}\right\rangle$ are eigenfunctions of $H_{\mathrm{BCS}}$. The first and second ones are the ground state functions of even systems with $N$ and $N+2$ particles, respectively. The latter, $\left|(N+1)_{t}\right\rangle$, is a $s=1$ function with an odd particle in state $t$. The energies $E(N), E(N+2)$ and $E_{t}(N+1)$ are corresponding eigenvalues. The system of equations becomes closed if the first assumption of the SPA comes into force, i.e. if the pair transfer matrix element (PTME) $\left\langle N+2\left|P^{\dagger}\right| N\right\rangle$ and $\langle N|P| N+2\rangle$ are many times more than $\left\langle(N+2)_{t}\left|P^{\dagger}\right| N\right\rangle$ and $\left\langle(N)_{t}|P| N+2\right\rangle$ where $\left|(N+2)_{t}\right\rangle$ and $\left|(N)_{t}\right\rangle$ are excited $s=0$ states. Therefore in expansion of products $a_{\bar{t}} P^{\dagger}$ and $a_{t}^{\dagger} P$ in intermediate states only one term is kept

$$
\begin{gathered}
\left\langle(N+1)_{t}\left|a_{t}^{\dagger} P\right| N+2\right\rangle=\left\langle(N+1)_{t}\left|a_{t}^{\dagger}\right| N\right\rangle \Delta^{(+)}, \\
\left\langle(N+1)_{t}\left|a_{\bar{t}} P^{\dagger}\right| N\right\rangle=\left\langle(N+1)_{t}\left|a_{\bar{t}}\right| N+2\right\rangle\left(\Delta^{(+)}\right)^{\dagger} .
\end{gathered}
$$

Repeating the same arguments as applied to $\left\langle(N-1)_{t}\left|a_{t}^{\dagger}\right| N-2\right\rangle$ and $\left\langle(N-1)_{t}\left|a_{\bar{t}}\right| N\right\rangle$ one comes to equations similar to (9) and (10) but instead of $\Delta^{(+)}$there will stand $\Delta^{(-)}$

$$
\Delta^{(+)}=g\langle N|P| N+2\rangle, \Delta^{(-)}=g\langle N-2|P| N\rangle .
$$

It is convenient to count out single-particle energies from the chemical potential which can be introduced so that at $N \gg 1$ it would be equal to $\partial E / \partial N$

$$
\lambda^{( \pm)}= \pm[E(N \pm 2)-E(N)] / 2 .
$$

The notations $\Delta^{( \pm)}$and $\lambda^{( \pm)}$are borrowed from [4].

After introducing $\lambda$ in Eqs. (7) and (8) the pairing energy $e_{t}=E_{t}(N+1)-[E(N+2)+E(N)] / 2$ appears quite naturally. We reserve for $e_{t}$ the usual name of quasiparticle energy since the differences of $e_{t}$ determine the low-lying part of spectra in odd nuclei.

$$
\begin{gathered}
E_{t}(N+1)-E(N)-\varepsilon_{t}=e_{t}-\left(\varepsilon_{t}-\lambda^{(+)}\right), \\
E_{t}(N+1)-E(N+2)+\varepsilon_{t}=e_{t}+\left(\varepsilon_{t}-\lambda^{(+)}\right) .
\end{gathered}
$$

Then Eqs. (7) and (8) with taking into account Eqs. (9), (13), (14) gain the form

$$
\begin{gathered}
{\left[e_{t}-\left(\varepsilon_{t}-\lambda^{(+)}\right)+g\right]\left\langle(N+1)_{t}\left|a_{t}^{\dagger}\right| N\right\rangle=} \\
=-\left\langle(N+1)_{t}\left|a_{\bar{t}}\right| N+2\right\rangle\left(\Delta^{(+)}\right)^{\dagger}, \\
{\left[e_{t}+\left(\varepsilon_{t}-\lambda^{(+)}\right)\right]\left\langle(N+1)_{t}\left|a_{\bar{t}}\right| N+2\right\rangle=} \\
=-\left\langle(N+1)_{t}\left|a_{t}^{\dagger}\right| N\right\rangle \Delta^{(+)} .
\end{gathered}
$$

The determinant of the system (15), (16) is to be equal to zero

$$
\begin{gathered}
{\left[e_{t}-\left(\varepsilon_{t}-\lambda^{(+)}\right)+g\right] \cdot\left[e_{t}+\left(\varepsilon_{t}-\lambda^{(+)}\right)\right]-\left|\Delta^{(+)}\right|^{2}=0,} \\
e_{t}=-\frac{g}{2}+\sqrt{\left(\varepsilon_{t}-\lambda^{(+)}-\frac{g}{2}\right)^{2}+\left|\Delta^{(+)}\right|^{2}} .
\end{gathered}
$$

As the pair energy is always positive the radical in Eq. (17) stands with sign plus.

There is a model in which SPA works quite exactly. This is the quasispin model [7] or the single shell model, $j+\frac{1}{2}=\Omega$, in which all single-particle energies are equal to zero and there exist no excited $s=0$ states. If in this model $\Delta^{( \pm)}$and $\lambda^{( \pm)}$are calculated in accordance with their definitions, Eqs. (11), (12), then a quasiparticle energy given by Eq. (17) is identical with the exact value of this quantity and equal to $g \Omega / 2$.

Changing the count points $\tilde{e}_{t} \rightarrow e_{t}+\frac{g}{2}, \tilde{\lambda} \rightarrow \lambda+\frac{g}{2}$ that is absolutely unessential for bulk systems in which $g \sim N^{-1}$ and $N \sim 10^{23}$ one obtains the standard quasiparticle energy (4).

For finite systems the term $g / 2$ in Eq. (17) can be modified at more exact calculations allowing for excited $s=0$ states. The impact of such states on $e_{t}$ can be traced for two simple examples. The first is the case $t \gg F$ ( $F$ is the Fermi level), the second corresponds to $t \ll F$, i.e. both cases deal with inequality $\left|\varepsilon_{t}-\lambda\right| \gg \Delta$. To correct $e_{t}$ in these cases additional excited states are taken into consideration: $a_{t}^{\dagger} a_{\bar{t}}^{\dagger}|N\rangle$ for $t \gg F$ and $a_{\bar{t}} a_{t}|N+2\rangle$ for $t \ll F$. These states are virtually orthonormal as for $t \gg F$ level $t$ is almost empty in $|N\rangle$ and $|N+2\rangle$ states: $a_{\bar{t}} a_{t}|N+2\rangle \simeq a_{\bar{t}} a_{t}|N\rangle \simeq 0$, $\left\langle N\left|1-\hat{n}_{t}\right| N\right\rangle \simeq 1$. For $t \ll F$ level $t$ is almost completely occupied in $|N+2\rangle$ and $|N\rangle$ and so $a_{t}^{\dagger} a_{t}^{\dagger}|N\rangle \simeq a_{t}^{\dagger} a_{t}^{\dagger}|N+2\rangle \simeq 0$, $\left\langle N+2\left|\hat{n}_{t}-1\right| N+2\right\rangle \simeq 1$. Now, insertion of $a_{t}^{\dagger} a_{\hat{t}}^{\dagger}|N\rangle$ as an additional intermediate state at $t \gg F$ in Eq. (7) equally with 
$|N+2\rangle$, and employment in Eq. (8) state $|N\rangle$ as the only intermediate state, and vice versa, $a_{\bar{t}} a_{t}|N+2\rangle$ at $t \ll F$ in Eq. (8) and the only state $|N+2\rangle$ in Eq. (7) result in

$$
\begin{gathered}
t \gg F, e_{t}=\sqrt{\left(\varepsilon_{t}-\lambda^{(+)}\right)^{2}+\left|\Delta^{(+)}\right|^{2}} ; \\
t \ll F, e_{t}=\sqrt{\left(\varepsilon_{t}-\lambda^{(+)}-g\right)^{2}+\left|\Delta^{(+)}\right|^{2}} .
\end{gathered}
$$

Since Eqs. (15), (16) are homogeneous they do not allow to found the absolute values of matrix elements $\left\langle(N+1)_{t}\left|a_{t}^{\dagger}\right| N\right\rangle$ and $\left\langle(N+1)_{t}\left|a_{\bar{t}}\right| N+2\right\rangle$. Nevertheless their ratio can be compared with the result of the simplified quasiparticle theory. If one assumes that $\left|(N+1)_{t}\right\rangle=\alpha_{t}^{\dagger}|0\rangle_{B}$ and $|N\rangle \simeq|N+2\rangle=|0\rangle_{B}$, where $|0\rangle_{B}$ is the Bogoliubov quasiparticle vacuum, then

$$
\left(\frac{\left\langle(N+1)_{t}\left|a_{\bar{t}}\right| N+2\right\rangle}{\left\langle(N+1)_{t}\left|a_{t}^{\dagger}\right| N\right\rangle}\right)_{\mathrm{BCS}} \simeq-\frac{v_{t}}{u_{t}}=-\frac{\left(e_{t}\right)_{\mathrm{BCS}}-\tilde{\varepsilon}_{t}}{\Delta} .
$$

Whilst the same ratio in SPA gains the form (as followed from Eq. (15))

$$
\left(\frac{\left\langle(N+1)_{t}\left|a_{\bar{t}}\right| N+2\right\rangle}{\left\langle(N+1)_{t}\left|a_{t}^{\dagger}\right| N\right\rangle}\right)_{\mathrm{SPA}}=-\frac{e_{t}-\left(\varepsilon_{t}-\lambda^{(+)}\right)+g}{\Delta^{(+)}} .
$$

Thus, the similarity of these quantities, i.e. Eqs. (20) and (21), in BCS and SPA is evident.

\section{Equations for $\operatorname{PTME}(\Delta)$ and chemical potential $(\lambda)$}

Equations (11) and (12) define $\Delta^{( \pm)}$and $\lambda^{( \pm)}$but give no way to count these quantities. To establish equations for $\Delta$ and $\lambda$ we make use the second assumption of the SPA, i.e. we assume that $a_{t}^{\dagger}|N\rangle$ or $a_{\bar{t}}|N+2\rangle$ are proportional to $\left|(N+1)_{t}\right\rangle-$ the eigenfunction of $H_{\mathrm{BCS}}$ with eigenvalue $E_{t}(N+1)$ (this is a true statement in the quasispin model):

$$
\left\langle N+2\left|a_{t}^{\dagger}\left[H_{\mathrm{BCS}}-E_{t}(N+1)\right] a_{\bar{t}}^{\dagger}\right| N\right\rangle=0 .
$$

Whence it follows that

$$
\begin{gathered}
\left\langle N+2\left|\left[H_{\mathrm{BCS}}-E_{t}(N+1)\right] a_{t}^{\dagger} a_{\bar{t}}^{\dagger}+\left[a_{t}^{\dagger}, H_{\mathrm{BCS}}\right] a_{t}^{\dagger}\right| N\right\rangle \\
=\left[E(N+2)-E_{t}(N+1)-\varepsilon_{t}\right]\left\langle N+2\left|a_{t}^{\dagger} a_{t}^{\dagger}\right| N\right\rangle \\
+\left(\Delta^{(+)}\right)^{\dagger}\left\langle N\left|1-\frac{1}{2} \hat{n}_{t}\right| N\right\rangle=0, \\
E_{t}(N+1)+\varepsilon_{t}-E(N+2)=e_{t}+\left(\varepsilon_{t}-\lambda^{(+)}\right) .
\end{gathered}
$$

In (23), the expectation value $\left\langle N\left|a_{t}^{\dagger} a_{t}\right| N\right\rangle$ is supposed to be equal to $\frac{1}{2}\left\langle N\left|\hat{n}_{t}\right| N\right\rangle$ by virtue of the time-invariance of $|N\rangle$ $\left(|N\rangle\right.$ is the ground state function with spin and parity $\left.0^{+}\right)$.

The second equation to find $\Delta$ and $\lambda$ exploits only the predominance of PTME between ground states (the first assumption of SPA)

$$
\begin{aligned}
{[E(N+2)-E(N)]\left\langle N+2\left|a_{t}^{\dagger} a_{t}^{\dagger}\right| N\right\rangle } \\
=\left\langle N+2\left|\left[H_{\mathrm{BCS}}, a_{t}^{\dagger} a_{\bar{t}}^{\dagger}\right]\right| N\right\rangle .
\end{aligned}
$$

Calculation of commutator in this equation gives

$$
2\left(\varepsilon_{t}-\lambda^{(+)}\right)\left\langle N+2\left|a_{t}^{\dagger} a_{\bar{t}}^{\dagger}\right| N\right\rangle=\left(\Delta^{(+)}\right)^{\dagger}\left\langle N\left|1-\hat{n}_{t}\right| N\right\rangle .
$$

Linear superpositions of Eqs. (23) and (25) lead to

$$
\begin{gathered}
\left\langle N+2\left|a_{t}^{\dagger} a_{t}^{\dagger}\right| N\right\rangle e_{t}=\frac{1}{2}\left(\Delta^{(+)}\right)^{\dagger}, \\
\left\langle N\left|\hat{n}_{t}\right| N\right\rangle=1-\frac{\varepsilon_{t}-\lambda^{(+)}}{e_{t}} .
\end{gathered}
$$

Recollecting the definitions of $\Delta^{(+)}$, Eq. (10), $P^{\dagger}$ and $\hat{n}_{t}$, Eq. (1), one obtains

$$
\begin{gathered}
\left(\Delta^{(+)}\right)^{\dagger}=g \sum_{t}\left\langle N+2\left|a_{t}^{\dagger} a_{t}^{\dagger}\right| N\right\rangle=\frac{g}{2} \sum_{t} \frac{\left(\Delta^{(+)}\right)^{\dagger}}{e_{t}}, \\
\frac{2}{g}=\sum_{t} \frac{1}{e_{t}} \\
N=\sum_{t}\left\langle N\left|\hat{n}_{t}\right| N\right\rangle=\sum_{t}\left[1-\frac{\varepsilon_{t}-\lambda^{(+)}}{e_{t}}\right] .
\end{gathered}
$$

Comparison of Eqs. (28) and (29) with the basic equations of the QM, Eq. (5), shows that SPA comes to the same equations for $\lambda$ and $\Delta$ as the QM (distinguishing in definitions of $\Delta, \lambda$ and $e_{t}$ ) though SPA employs neither the BCS function nor the Bogoliubov transformation. As shown in [3], the SPA gives the 4-th order equation for the low-lying $s=2$ state energy $\omega_{12}=E_{12}(N)-E(N)$, "1,2" are single-particle states:

$$
\begin{gathered}
\omega_{12}-2(R-g)-\omega_{12}^{-1}\left(\varepsilon_{2}-\varepsilon_{1}\right)^{2}=0, R=R^{(+)}+R^{(-)}, \\
R^{(+)}=\left|\Delta^{(+)}\right|^{2}\left[\omega_{12}-2 \lambda^{(+)}+\left(\varepsilon_{1}+\varepsilon_{2}\right)\right]^{-1}, \\
R^{(-)}=\left|\Delta^{(-)}\right|^{2}\left[\omega_{12}+2 \lambda^{(-)}-\left(\varepsilon_{1}+\varepsilon_{2}\right)+2 g\right]^{-1},
\end{gathered}
$$

where $\omega_{12}$ is the maximum real solution to Eq. (30). In the exactly solvable quasispin model all quantities entering into Eq. (30) are easily calculated

$$
\begin{gathered}
\Delta^{(+)}=\Delta(N)=0.5 g[(2 \Omega-N)(N+2)]^{\frac{1}{2}} ; \\
\lambda^{(+)}=\lambda(N)=-0.5 g(\Omega-N) ; \\
\Delta^{(-)}=\Delta(N-2) ; \quad \lambda^{(-)}=\lambda(N-2),
\end{gathered}
$$

where $N$ is the particle number in the shell. In this model all $\varepsilon_{i}=0$, therefore Eq. (30) reduces to the third-order equation. The maximum value of solutions to this equation is $g \Omega$ and this is identical with the exact solution for $s=2$ state in this model.

\section{Results of calculations}

The calculations implemented in accordance with Eqs. (5), (17), (28), (29) confirm, as a matter of fact, the similarity of results following from the SPA and those given by QM. As an example, we present theoretical results for protons in ${ }_{64}^{148} \mathrm{Gd}_{84}$. We compare results of QM and SPA with the exact solutions to $H_{\mathrm{BCS}}$, Eq. (1), but not with experimental spectra since for such comparison it would be necessary 
Table 1. Proton energies for $s=1$ states of ${ }_{65}^{149} \mathrm{~Tb}_{84}$. Energies $(\mathrm{MeV})$ are counted from the energy of $1 h_{11 / 2}$ state.

\begin{tabular}{llll}
\hline$s=1$ state & exact & BCS & SPA \\
\hline $1 h_{11 / 2}$ & 0.000 & 0.000 & 0.000 \\
$2 d_{5 / 2}$ & 0.006 & -0.384 & -0.173 \\
$2 d_{3 / 2}$ & 0.016 & 0.014 & 0.013 \\
$3 s_{1 / 2}$ & 0.260 & 0.230 & 0.204 \\
$1 g_{7 / 2}$ & 1.044 & 0.470 & 0.699 \\
$1 g_{9 / 2}$ & 4.740 & 4.107 & 4.310 \\
$2 p_{1 / 2}$ & 6.204 & 5.240 & 5.439 \\
$2 p_{3 / 2}$ & 6.855 & 6.221 & 6.417 \\
$1 f_{5 / 2}$ & 8.739 & 8.106 & 8.298 \\
\hline
\end{tabular}

Table 2. Two-proton energies (MeV) for $s=2$ states of ${ }_{64}^{148} \mathrm{Gd}_{84}$. $\Delta_{\mathrm{BCS}}=1.355 \mathrm{MeV} ; \Delta_{\mathrm{SPA}}^{(+)}=1.358 \mathrm{MeV}$.

\begin{tabular}{llll}
\hline$s=2$ configuration & exact & BCS 2qp. & SPA \\
\hline $2 d_{5 / 2}, 2 d_{3 / 2}$ & 3.179 & 3.370 & 3.488 \\
$\left(2 d_{5 / 2}\right)^{2}$ & 3.185 & 2.972 & 3.173 \\
$2 d_{5 / 2}, 3 s_{1 / 2}$ & 3.442 & 3.586 & 3.697 \\
$\left(1 h_{11 / 2}\right)^{2}$ & 4.048 & 3.740 & 4.003 \\
$\left(2 d_{3 / 2}\right)^{2}$ & 4.084 & 4.224 & 4.033 \\
$1 g_{7 / 2}, 2 d_{3 / 2}$ & 4.243 & 3.984 & 4.262 \\
$2 d_{5 / 2}, 1 g_{7 / 2}$ & 4.253 & 3.826 & 4.036 \\
$3 s_{1 / 2}, 2 d_{3 / 2}$ & 4.347 & 3.984 & 4.255 \\
$1 g_{7 / 2}, 3 s_{1 / 2}$ & 4.496 & 4.440 & 4.461 \\
$\left(1 g_{7 / 2}\right)^{2}$ & 5.312 & 4.680 & 4.915 \\
\hline
\end{tabular}

to take into account interactions with phonon excitations (pair-, quadrupole- vibrations etc).

As the neutron number in ${ }^{148} \mathrm{Gd}$ is close to the magic one this nucleus is spherical. Therefore we use for protons the spherical Saxon-Woods potential with a standard set of parameters [8]. In the pairing problem we take into consideration 9 levels: from $1 f_{5 / 2}$ up to $3 s_{1 / 2}$. The Fermi level corresponds to the subshell $2 d_{5 / 2}$. The pairing strength is equal to $0.2 \mathrm{MeV}$. The BCS and SPA chemical potentials are about identical: $\lambda_{\mathrm{BCS}}=-4.38 \mathrm{MeV}$, $\lambda_{\mathrm{SPA}}^{(+)}=-4.30 \mathrm{MeV}$. This difference is caused by different definitions of the quasiparticle energy in the QM, Eq. (4), and in the SPA, Eq. (17). Several levels (the lowest ones at a fixed quantum numbers of an odd proton) of the theoretical spectrum are shown in Table 1 for the neighbouring nucleus ${ }_{65}^{149} \mathrm{~Tb}_{84}$. The exact energy spectrum of $s=1$ states with fixed $n l j$, counted from the lowest such state, nearly repeats the exact spectrum of $s=0$ states (see Fig. 1). This can be evidence of weak mutual influence of $s=1$ states and indirectly confirms the second hypothesis of SPA $\left(|N+1\rangle_{t} \sim a_{t}^{\dagger}|N\rangle\right)$.

Energy spectra of low-lying one-quasiparticle states, $s=1$, Eq. (17), and two-quasiparticle ones, $s=2$, Eq. (30), are given in Tables 1,2, respectively, in comparison with the corresponding differences of eigenvalues of $H_{\mathrm{BCS}}$ which are denoted as "exact". The SPA energies are somewhat closer to exact ones but it is hardly possible to say that SPA gives a better description than QM. Both SPA and QM compress spectra at energies $>4 \mathrm{MeV}$, but deviations from the exact values are less than $2 g$.

\section{Conclusion}

The main result obtained by the Green function method in Ref. [3] or by the equations-of-motion method considered in this paper is that the basic BCS equations can be established without involving the BCS function or the Bogoliubov transformation from particles to quasiparticles. Thereby these equations (at corresponding redefinition of the chemical potential, quasiparticle energy and gap parameter) are not connected with fluctuations of the particle number. Hence it follows that improvement of the energy description could be attained by means of taking into account various phonon excitations. The mechanism for that is elaborated in the quasiparticle theory, however, for the theory in which the particle number is fixed such mechanism requires a special formulation.

\section{Acknowledgements}

This work was supported by the St.-Petersburg State University grant, project No. 11.38.648.2013.

\section{References}

[1] V.G. Soloviev, Theory of Atomic Nuclei: Quasiparticles and Phonons (Institute of Physics, Bristol and Philadelphia, 1992)

[2] J. von Delft and D.C. Ralph, Phys. Rep. 345, 61 (2001)

[3] A.K. Vlasnikov, A.V. Lunev, V.M. Mikhailov, Bull. Russ. Acad. Sci. Phys. 75, 569 (2011)

[4] L.P. Gorkov, Sov. Phys. JETP 7, 505 (1958)

[5] A. Klein, Prog. Part. Nucl. Phys. 10, 39 (1983)

[6] F. Andreozzi, A. Covello, A. Gargano, A. Porrino, Phys. Rev. C 37, 2228 (1988)

[7] A.K. Kerman, Ann. Phys. 12, 300 (1961)

[8] V.I. Isakov, K.I. Erokhina, H. Mach, M. SanchezVega, B. Fogelberg, Eur. Phys. J. A 14, 29 (2002)

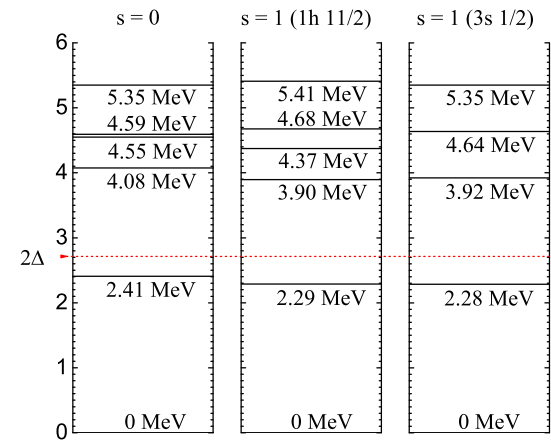

Figure 1. (Color online) The canonical energy spectra of $s=0$ $\left(0^{+}\right.$, ground) and $s=1$ (with one unpaired particle on $1 h_{11 / 2}$ or $3 s_{1 / 2}$ levels) states. 